\title{
Realidad y ficción en Utopía: un acercamiento a la obra a partir del concepto de experimento mental*
}

\author{
Reality and fiction in Utopia: an approach to this work from the concept of \\ thought experiment.
}

\author{
Por: Huespe, Mayra* \\ Universidad Nacional del Litoral \\ Santa Fe, Argentina \\ E-mail: mayrahuespe@hotmail.com
}

Fecha de recepción: 17/03/2021

Fecha de aprobación: 25/04/2021

DOI: http://dx.doi.org/10.30972/ach.065602

\section{Resumen}

El objetivo del presente trabajo es proponer un acercamiento a la obra Utopía utilizando como clave de lectura el concepto de experimento mental. Para ello, en un primer momento, 1-exponemos una serie de dificultes que emergen de la obra relativas a los límites entre la realidad y la ficción. Específicamente, nos centramos en la problemática ampliamente tratada de desglosar a Moro-autor de Moro-personaje. Para ello, abordamos uno de los pasajes cruciales en donde la voz creadora y la voz creada parecen entrar en un conflicto irresoluble: aquel en donde la personificación del autor asume no estar del todo convencido respecto de la tesis central defendida a lo largo de toda la obra. La motivación de esta sección es presentar la relación entre realidad y ficción en Utopía en los términos de una problemática abierta, sobre la cual aún hay mucho por decir. En un tercer momento, 2-exponemos la definición del concepto de experimento mental al que recurrimos en nuestro trabajo. Por último, 3-

\footnotetext{
* Trabajo realizado en el marco del Seminario I de la Licenciatura en Filosofía de la Facultad de Humanidades y Ciencias de la Universidad Nacional del Litoral.

* Estudiante avanzada de la Licenciatura en Filosofía de la Facultad de Humanidades y Ciencias de la Universidad Nacional del Litoral (FHUC-UNL).
} 
desarrollamos nuestra propuesta central: utilizar el concepto de experimento mental como clave de lectura de la obra. La idea es mostrar cómo el concepto seleccionado, al articularse con una concepción polifónica de la obra, logra iluminar aquellos pasajes en donde los límites entra la realidad y la ficción parecen entrar en conflicto.

Palabras claves: Utopía - Tomas Moro - Experimento Mental - Häggqvist - Realidad y Ficción.

\section{Abstract}

The paper aim is to propose a new approach to Utopia using the concept of a thought experiment like a key for reading. To get this objective, 1-we first expose the difficulties that emerge from Utopia related to limits between reality and fiction. Specifically, we focus on the widely discussed problem of distinguishing Morecharacter ideas from More-author ideas. We focus on a crucial passage from Utopia where the creator and the created voices seem to enter into an irresolvable conflict: when the author's personification says that he is not convinced about the central thesis defended throughout the entire book by Rafael. This section of de paper is motivated to reveal the relationship between reality and fiction in terms of an open problem, and about which there is still much to say. Afterward, 2-we expose the definition of the thought experiment used in this paper. Finally, 3-we develop our primary aim: to use the concept of a thought experiment as a key for reading to Utopia. The idea is to show how the concept of thought experiment can illuminate those passages where the limits between reality and fiction seem to get in conflict.

Key words: Thomas More-Utopia-Thought Experiment-Häggqvist-Reality and Fiction 
Abril 2021.

ISSN 2344-9934

\section{Cómo citar este artículo:}

APA: Huespe, M. (2021). Realidad y ficción en Utopía: un acercamiento a la obra a partir del concepto de experimento mental. Acheronta, № 6, 76-94. Recuperado de: (agregar dirección web)

\section{1-Realidad y ficción en Utopía}

Una extraña sensación de incomodidad asecha al lector de Utopía que trata de distinguir los límites que separan los escenarios reales de los ficticios. A lo largo de toda la obra, Moro juega con este antagonismo: personajes reales dialogando con personajes ficticios, lugares reales utilizados para establecer la ubicación por contigüidad de lugares ilusorios, ciudades imaginarias que nos permiten conocer las causas de los mayores males que asedian en nuestras ciudades reales. En este sentido, un problema fundamental que interpela al lector en su recorrido por la obra es la relación entre realidad y ficción.

Un requerimiento para aceptar las conclusiones de la obra es leer este antagonismo en los términos de una fusión: "Utopía es aquel lugar imaginario por el que se debe, imaginariamente, pasar para tomar conciencia de las causas del mal profundo que sufren Inglaterra y Europa" (Moro, 1987, p. 35). A partir de la construcción racional de una república ficticia, Moro extrae una serie de conclusiones relativas a la situación social y política de Europa. La Isla Utopía parece ser una especia de herramienta cognitiva a partir de la cual el lector obtiene nuevos conocimientos de índole social y político. Una construcción racional imaginaria que nos permite avanzar en nuestro conocimiento relativo a la situación política de Europa en el siglo XVI.

Ahora bien, entender este antagonismo entre realidad y ficción en los términos de una fusión no es un posicionamiento que carezca de problemas. Por ejemplo, uno de los temas más discutidos por los especialistas es el posicionamiento que Moro como autor toma respecto de los diversos temas políticos y sociales abordados en Utopía. Al recorrer la obra, el lector reconoce una duplicidad subyacente: Moro es personaje y autor, realidad y ficción, la voz creadora y la voz creada. Desglosar las opiniones del autor de las proferidas por su personificación no es una tarea fácil. En 
efecto, actualmente no hay consenso entre los comentaristas respecto de este tópico. Un punto crucial para esta discusión es uno de los pasajes finales en donde la personificación de Moro vuelve a retomar la primera objeción que esgrimió contra la propiedad privada. Una de las ideas principales que se despliega a lo largo de toda la obra es la concepción de la propiedad privada como la causa del mal profundo que asecha a Inglaterra. Sin embargo, en los pasajes finales de la obra, nada menos que la personificación del autor reconoce que no está del todo convencido respecto de la tesis central. En este punto, la duplicidad subyacente a toda la obra se vuelve manifiesta para el lector: voz creadora y voz creada entran en un conflicto aparentemente irresoluble:

Cuando Rafael hubo relatado estas cosas, aunque me venían a la mente no pocas que me parecían muy absurdamente instituidas en las costumbres y leyes de este pueblo, no solo sobre la manera de hacer la guerra, y sobre las cuestiones divinas, y sobre la religión, y sobre sus otras instituciones además, pero sobre todo en lo que es el máximo fundamento de toda institución, a saber, en la vida y el sustento común, sin ningún intercambio de dinero, cosa sola esta por la que se destruye radicalmente toda la nobleza, magnificencia, esplendor, majestad, como es pública opinión, verdaderas glorias y ornamentos de la república[...] Entretanto, igual que no puedo asentir a todo lo dicho por un hombre, de otra manera, sin discusiones, muy erudito y muy sabedor a la vez de las cosas humanas, así confieso fácilmente que hay muchísimas cosas en la república utopiense que, a la verdad, en nuestras ciudades, más estaría yo en desear que en esperar (Moro, 1987, pp. 132 y 133).

Los comentaristas han tomado diversos caminos para dar una explicación del movimiento peculiar que realiza Moro en este pasaje. Por ejemplo, Sylvester (1977) opta por explicarlo sosteniendo la tesis de que las ideas defendidas por Moropersonaje no corresponden con las de Moro-autor. De modo más general, el comentarista propone una lectura de la obra haciendo énfasis en su carácter dialógico: 
Thus the order in which we read the work, which represents More's final intention, reverses the order in which he conceived it; the genetics of composition have been displaced by a measured rethinking and reassessment; what was once an autonomous monologue has been set in a dialogic framework. Perhaps our own interpretations of the work as a whole should be modelled on this process. Utopia, as we have it, begs us to continue the discussion, to confront Hythlodaeus for ourselves so that, as Thomas More puts it at the end of Book II, we may have another chance "to think about these matters more deeply and to talk them over with him more fully" (245) (Sylvester, 1977, p. 277).

Siguiendo un camino similar y retomando a Blaim (1982), Sánchez (2011) opta por hacer una lectura de la obra en clave polifónica. Desde esta óptica, el comentarista proporciona un panorama de Utopía como una exposición de diversos puntos de vista que nunca convergen y que se hallan en continua incidencia recíproca. Dice el autor al respecto:

Hablando de Utopía, el humanista refleja, de alguna manera, los problemas con los que tendría que enfrentarse cualquier concepción y búsqueda de sentido del mundo que le era contemporáneo. Pero, como todo intento de dar una imagen cabal del espacio vital que se ocupa, su visión no es más que una propuesta personal; y en este caso, es una propuesta hecha con la consciencia de que sólo puede tratarse de una propuesta personal, y de que toda concepción de un mundo se encuentra originalmente enfrentada a otras simultáneas que son siempre susceptibles de influir en el sentido, aplicabilidad, vigencia y alcances de dicha concepción; o, en otras palabras, que se da dentro de un corpus dialogístico de agencias que interactúan unas con otras (Sánchez, 2011, p. 37).

En lo que sigue retomamos y articulamos la lectura de Sánchez y Blaim con nuestro objetivo: proponer acercamiento a la obra Utopía utilizando como clave de lectura el concepto de experimento mental de Häggqvist (2009). Para ello, exponemos 
en la siguiente sección la definición del concepto de experimento mental a la que recurrimos en nuestro trabajo, para luego desarrollar la propuesta central: utilizar la definición en cuestión como clave de lectura de la obra. La idea es mostrar cómo el concepto seleccionado de experimento mental, al articularse con una concepción polifónica de la obra, como la defendida por Sánchez y Blaim, logra iluminar aquellos pasajes en donde realidad y ficción parecen entrar en conflicto.

\section{2-Experimentos mentales en filosofía}

Para introducirnos sin mayores preámbulos en la vastísima área de los experimentos mentales, podemos definirlos de modo muy general como dispositivos de la imaginación empelados para diversos fines (Brown y Fehige, 1996, p. 1). En nuestro trabajo nos interesan especialmente los experimentos mentales filosóficos que nos permiten investigar la realidad política y social a través de la construcción de situaciones contrafáticas. Un desafío central en las discusiones que se vienen dando en el ámbito filosófico respecto de este concepto es el que concierne su carácter epistemológico. Las preguntas centrales en estas discusiones atañen a la posibilidad de conocer aspectos de la realidad únicamente a partir de situaciones contrafáticas imaginadas:

Looking at the development of the discussion about thought experiments over the past thirty years, it is fair to say that thought experiments were primarily an important topic in the philosophy of science and the philosophy of philosophy ("metaphilosophy"), before the scope widened up at a later point. There is a simple reason for that path. At the core of the discussion sits a relatively simple epistemological challenge that is presented in a particularly powerful manner by numerous thought experiments that the history of science has to offer. They suggest that we can learn about the real world by virtue of merely thinking about imagined scenarios. But how can we learn about reality (if we can at all), just by thinking in such a way? Are there really thought experiments that enable us to acquire new knowledge about nature without new empirical data? If so, where does the new information come from 
if not from contact with the realm of investigation under consideration in an imagined scenario? (Brown y Fehige, 1996, p. 2).

Se han dado diversas respuestas a todas estas preguntas. Dentro de las principales corrientes encontramos la explicación basada en la intuición (Brown 1991), el constructivismo conceptual (Van Dyck, 2003), la visión argumental (Norton, 1991), entre otras. En el presente trabajo retomamos la propuesta que desarrolla Haägqvist (1996 y 2009). Para el autor, los experimentos mentales son casos hipotéticos que funcionan, efectivamente, como experimentos, en el sentido de que aspiran a testear hipótesis o teorías (Häggqvist, 2009, p. 57). En lo que sigue exponemos los elementos centrales de su concepción.

Una opción para justificar epistémicamente los experimentos mentales, sin tener que postular la existencia de algún tipo de facultad especial no empírica, es identificándolos con los argumentos. El autor rechaza la propuesta de identificación total, sin embargo, considera que la visión argumental captura algunas cuestiones importantes. En primer lugar, los experimentos mentales, al igual que los experimentos ordinarios, funcionan únicamente a través de su conexión con los argumentos. Su utilidad radica en su potencial para verificar o falsear ciertas hipótesis o teorías. A su vez, el valor de verdad de tales hipótesis o teorías es evaluado mediante argumentos. Sin embargo, los experimentos, tanto mentales como ordinarios, no son reducibles a argumentos y carecen de valores de verdad. Para explicar tal conexión evitando la propuesta de la identificación total, el autor formula una relación del tipo causal, en el siguiente sentido:

I suggest that it is causal and, in broad stroke, this: events taking place in a laboratory experiment causes observers in the lab to hold certain observation statements true, which may then be employed in arguments concerning the theory to be tested. And performing a thought experiment causes thought experimenters - inventor or audience - to hold certain non-observational statements true, which may subsequently be employed in arguments concerning the theory to be tested. Thus both experiments and thought 
experiments are conducted to generate (acceptance of) premises for certain arguments concerning some theory or hypothesis (Häggqvist, 2009, p. 62).

Desde la propuesta de Häggqvist, por lo tanto, la conexión entre argumentos y experimentos mentales se explica, en primer lugar, por una relación del tipo causal: los experimentos mentales producen la creencia en la verdad o falsedad de ciertas afirmaciones que luego son empleadas en argumentos que determina el valor de verdad de ciertas teorías o hipótesis. En segundo lugar, tal conexión se explica por el hecho de que los experimentos se transmiten y reproducen de forma lingüística. En consecuencia, pese a que no son reducibles a esquemas de argumento, sí mantienen una relación estrecha con algunos de ellos.

\section{3-El concepto de experimento metal como clave de lectura de Utopía}

En la sección anterior expusimos los rudimentos básicos de la concepción de Häggqvist respecto a los experimentos mentales. En lo que sigue, continuamos profundizando en su propuesta, pero aplicándola a nuestros intereses: proponer una lectura de la Isla Utopía en los términos de un experimento mental. Para ello, a-en un primer momento exponemos dos de los cuatro esquemas de argumento que, según Häggqvist, están relacionados con los experimentos mentales. Luego, b-mostramos en qué sentido se puede entender a la Isla Utopía como una premisa contrafáctica que forma parte de un argumento que instancia el primer esquema de Häggqvist. En un tercer momento, c-mostramos en qué sentido se puede comprender la respuesta problemática de Moro como la premisa de un argumento que instancia el segundo esquema Häggqvist. Por último, d- sostenemos que nuestra lectura descansa sobre una concepción polifónica de Utopía, en el sentido defendido por Sánchez y Blaim.

\section{3.a-Dos esquemas de argumento:}

Una de las principales críticas que se puede esgrimir contra las propuestas que postulan una relación estrecha entre los argumentos y los experimentos mentales es la diferencia que tiene ambos respecto de sus posibles conclusiones. Los experimentos mentales pueden tener diversas conclusiones, dependiendo de quién los interprete. Los argumentos, por el contrario, tiene una única conclusión correcta, más allá de sus 
Abril 2021.

ISSN 2344-9934

intérpretes. Häggqvist responde a esa crítica sosteniendo que las diversas conclusiones que se siguen de los experimentos mentales refieren a su potencial para ser leído a través de diversos esquemas de argumento.

Específicamente, el filósofo considera que se pueden relacionar a los experimentos mentales con cuatro esquemas de argumentos. El primer esquema que propone es central, debido a que responde a los intereses que tiene el/la experimentador/a mental. En otras palabras, la conclusión del primer esquema es aquella a la que deberíamos llegar en una situación ideal, en donde el experimento mental es exitoso. Pero hay otros esquemas que responden a las situaciones en donde el experimento no llega a buen puerto y, por lo tanto, la audiencia de el/la experimentador/a no logra arribar a la conclusión deseada. Tales casos se constituyen como instancias de los otros tres esquemas de argumento. Consideramos que en Utopia podemos ver reflejada los dos primeros esquemas de argumento. El primero de ellos lo reconocemos en los movimientos que realiza Rafael a lo largo de toda la obra; en efecto, este personaje utiliza a la descripción de la isla, entre otras cosas, como medio para defender sus ideas respecto de la propiedad privada. El segundo esquema lo vemos reflejado en el movimiento que realiza Moro-personaje en el último pasaje, cuando afirma que no se encuentra del todo convencido respecto de la tesis esgrimida por Rafael contra la propiedad privada.

El primer esquema de argumento busca explicitar el hecho de que, a partir de los experimentos mentales, ciertas tesis o teorías que antes eran aceptadas son falseadas. En este esquema la letra $\mathrm{C}$ refiere al escenario contrafáctica descripto por el experimento mental, $\mathrm{T}$ es la teoría o hipótesis testeada y $\mathrm{W}$ refiere a una afirmación con la cual se compromete la teoría o hipótesis testeada pero que, a su vez, es falsa en el escenario contrafáctica. 
(a) $1-\diamond C$

$$
2-\mathrm{T} \rightarrow(\mathrm{C} \square \rightarrow \mathrm{W})
$$

3- $\mathrm{C} \square \rightarrow \mathrm{W}$

4- ᄀТ

Según Häggqvist, este primer esquema logra capturar la progresión dialéctica de los experimentos mentales: "[...] first, a counterfactual scenario presented as possible is offered for contemplation; then the thesis under attack is held to be connected to a certain consequence in that scenario; whereupon the non-emergence of that consequence in that scenario is asserted" (Häggqvist, 2009, p. 63). A partir de un análisis de este primer esquema de argumento, queda claro cuál es la mayor ambición epistémica de los experimentos mentales: constituirse como situaciones contrafáticas que refutan algún aspecto de ciertas teorías al incluirse en instancias de $(\alpha)$.

El segundo esquema de argumento, como hemos explicitado antes, se constituye como intento fallido en donde el auditorio no llega a la conclusión esperada por el experimentador mental. En este segundo caso, se rechaza el reclamo del experimentador relativo a lo que debería ser el caso en la situación contrafáctica:

(B) 1- $\mathrm{T}$

2- $\diamond C$

3- $\mathrm{T} \rightarrow(\mathrm{C} \square \rightarrow \mathrm{W})$

4- $\diamond \mathrm{C} \wedge \mathrm{C} \square \rightarrow \mathrm{W}$

$$
\neg(\mathrm{C} \square \longrightarrow \neg \mathrm{W})
$$


La conclusión de este esquema implica:

La piedra angular es negar que haya algo malo en sostener que $\mathrm{W}$ es cierto en C. El nombre que el autor le adjudica a este esquema en el que defiende T es biting the bullet.

\section{3.b-Aplicación del primer esquema Häggqvist:}

Finalizando el Libro Primero, Rafael sostiene que para erradicar todos los males que asechan a las sociedades europeas es menester eliminar la propiedad privada:

Mi querido Moro (por decir lo que realmente pienso), me parece que dondequiera las posesiones son privadas, donde todos miden todas las cosas con el dinero, ahí apenas si podrá lograrse que con una república marchen las cosas justa o prósperamente, a no ser que seas de la opinión que se obra justamente donde todo lo mejor va a parar a los peores, o que la cosa marcha felizmente donde todo está repartido entre poquísimos, que ni siquiera están bien bajo todos los aspectos, estando los demás empero absolutamente en la miseria [...] El solo y único camino hacia el bienestar público está en declarar la comunidad de bienes, y esto no sé si se podrá guardar donde lo que posee cada uno es su propiedad[...] iTan firmemente estoy persuadido de que si no se suprime de raíz la propiedad no se puede distribuir los bienes según un criterio ecuánime y justo o disponer provechosamente los asuntos de los mortales! Sino que, si subsiste, subsistirá para la parte mayor y mejor con mucho de los hombres la ansiosa e ineluctable pesadumbre de la indigencia y de los infortunios (Moro, 1987, pp. 42 y 43).

En pocas palabras, Rafael mantiene que, para que haya bienestar público, es necesario la igualdad de condiciones entre todos los habitantes. Y sólo se logrará tal igualdad una vez que haya sido eliminada la propiedad privada. Si analizamos las palabras de Rafael con detenimiento, podremos ver que su propuesta tiene la forma de un condicional: si se elimina la propiedad privada, entonces habrá bienestar público. Frente a ello, las críticas centrales que la personificación del autor esgrime contra tal tesis son: 1-si eliminamos la propiedad privada, desaparece el incentivo para 
trabajar. En una situación así, la producción sería cada vez menor, lo que generaría escases que, a su vez, produciría a un estado de guerra de todos contra todos y 2 -sin propiedad privada no hay diferencia entre los hombres, jerarquías sociales, diferenciales de poder, orden. Ambas criticas pueden resumirse en la idea nuclear de que no es posible para las sociedades estar rectamente organizadas sin propiedad privada. Desde esta óptica, por tanto, el fundamente del orden social es la propiedad privada. Dice Moro-personaje al respecto:

-A mí, por el contrario -le dije yo-, me parece que no es posible vivir convenientemente donde todas las cosas son comunes. Porque ¿cómo puede haber un acopio suficiente de bienes si cada cual, puesto que no le urge el incentivo del lucro personal y la confianza en la laboriosidad ajena le vuelve indolente, se sustraerá al trabajo? Y si estimulados no obstante por la pobreza ni siquiera lo que hubiera logrado uno puede defenderlo como suyo de la mano de alguna ley, ¿no sería preciso habérselas con el asesinato continuo y la sedición? Sobre todo, después de suprimida la autoridad y reverencia de los magistrados, para lo que no puedo ni imaginarme qué cabida habría con hombres entre los que no media distinción ninguna (Moro, 1987, p. 44).

En este contexto, la descripción de la isla Utopía se presenta como la piedra angular en la defensa que propone Rafael de la eliminación de la propiedad privada. Al explicarle minuciosamente a sus oyentes las características sociopolíticas de la isla, Rafael no hace más que argumentar a favor de la posibilidad de concebir, o mejor dicho, de imaginar ${ }^{1}$, una sociedad rectamente organizada en donde no haya propiedad

\footnotetext{
${ }^{1}$ En este punto cobra especial relevación la última frase de la cita de Moro relativa a la imposibilidad de imaginar el respeto a la autoridad en una sociedad sin propiedad privada o, desde una perspectiva más general y siguiendo la idea defendida en el párrafo, la imposibilidad de imaginar el orden en una sociedad sin propiedad privada: "[...]sobre todo, después de suprimida la autoridad y reverencia de los magistrados, para lo que no puedo ni imaginarme qué cabida habría con hombres entre los que no media distinción ninguna." (Moro, 1987, p. 44) En la traducción que ofrece Prévost (1969) de este mismo pasaje queda aún más manifiesto el énfasis que está haciendo Moro-personaje en la imposibilidad de imaginar una rectamente organizada sin propiedad privada: "Eh bien, moi, dis-je, je pense tout le contraire. Jamais les hommes ne connaîtront l'aisance sous le régime de la communauté des biens. Par quels moyens, en effet, procurer des

biens en abondance, si chacun se dérobe au travail, comme c'est bien normal, puisque personne n'est aiguillonné par le souci de ses besoins personnels et que chacun, comptant sur l'activité d'autrui, s'abandonne à la paresse? Mais, comme la misère excite les esprits et qu'il ne sera plus possible de faire
} 
Abril 2021.

privada. En este sentido, a través de tal descripción, Rafael trata de refutar la tesis puesta en boca de Moro-personaje, según la cual el fundamento del orden social es la propiedad privada. Desde esta óptica, la descripción de Utopía se constituye como una premisa dentro de un argumento mayor desarrollado al largo de toda la obra. Tal argumento es una instancia del primer esquema propuesto por Häggqvist. Enfoquémonos en lo que sigue en analizar con mayor detalle la viabilidad de nuestra propuesta. En la aplicación del primer esquema de argumento, cada letra refiere a las siguientes afirmaciones:

C: Hay una Isla llamada Utopía en donde no hay propiedad privada.

T: el fundamento del orden social es la propiedad privada

W: ser una sociedad que carece de bienestar publico

La isla utopía es, por tanto, la situación contrafáctica que debemos imaginar para poder rechazar la hipótesis según la cual el fundamento de todo orden social es la propiedad privada. A su vez, ser una sociedad que carece de orden social es la afirmación que debería seguirse de la situación contrafáctica $C$, de ser verdadera la hipótesis T. El argumento, por tanto, queda de la siguiente forma:

( $\alpha$ ) P1: es posible que exista una Isla llamada Utopía en donde no hay $\quad(\diamond C)$. propiedad privada

P2: Si el fundamento de toda orden social es la propiedad privada, entonces, si en la Isla Utopía no hay propiedad privada, su sociedad carece de bienestar público $(\mathrm{T} \rightarrow(\mathrm{C} \square \rightarrow \mathrm{W}))$.

P3: Si en la Isla Utopía no hay propiedad privada, entonces su sociedad no $(\mathrm{C} \square \rightarrow \neg \mathrm{W})$.

appel à la loi pour protéger son bien, la société ne sombrera-t-elle pas fatalement et perpétuellement dans la sédition et le? En outre, si l'on fait disparaître l'autorité des magistrats et la crainte salutaire qu'ils inspirent, comment attribuer une place dans la société à des hommes entre qui n'existerait aucune différence de condition? C'est ce que je ne puis même imaginer." (Prévost, 1969, p. 442) 
carece de bienestar público

C4: Por lo tanto, el fundamento de todo orden social no es la propiedad privada $(\neg T)$.

3.c-Aplicación del segundo esquema de argumento propuesto por Häggqvist:

Como hemos visto al principio del trabajo, finalizado el Libro Segundo, Moro vuelve a esgrimir una crítica contra la eliminación de la propiedad privada. Este movimiento que deja perplejo al lector es aún un problema abierto para los comentaristas. En lo que sigue, tratamos de proporcionar una explicación original retomando, principalmente, tres autores: Sánchez, Blaim y Häggqvist. Tal explicación tiene dos momentos. El primero de ellos tiene como núcleo la idea que venimos desarrollado: la Isla Utopía debe endentarse en los términos de un experimento mental. El segundo momento gira en torno al carácter polifónico de la obra.

Entender a la Isla Utopía en los términos de un experimento mental en el sentido propuesto por Häggqvist nos proporciona algunas herramientas para iluminar el pasaje problemático que nos interesa. Como ya hemos explicado detalladamente, Häggqvist defiende que los experimentos mentales mantienen una estrecha relación con los argumentos. Específicamente, se constituyen como premisas de argumentos que buscan rechazar ciertas teorías o hipótesis. Los experimentos están relacionados con cuatro esquemas de argumento, y solo el primero responde a las intenciones del experimentador. Los otros tres casos se constituyen como esquemas fallidos, en donde el auditorio niega, por diversos caminos, la injerencia de la premisa contrafáctica en la determinación de la falsedad de T. Consideramos que, el esquema dos corresponde al camino que toma Moro-personaje para negar tal injerencia, es decir, para postular que la descripción de Utopía no logró convencerlo. De ser si, la forma del argumento es:

( $\beta$ ) P1: el fundamento de todo orden social es la propiedad privada (T).

P2: es posible que exista una Isla llamada Utopía en donde no hay $(\diamond \mathrm{C})$ 
Abril 2021.

ISSN 2344-9934

propiedad privada

P3: Si el fundamento de toda orden social es la propiedad privada, entonces, si en la Isla Utopía no hay propiedad privada, su sociedad carece de bienestar público $(\mathrm{T} \rightarrow(\mathrm{C} \square \rightarrow \mathrm{W}))$.

P4: es posible que exista una Isla llamada Utopía en donde no hay propiedad privada y, si en la Isla Utopía no hay propiedad privada, entonces $(\diamond \mathrm{C} \wedge \mathrm{C} \square \rightarrow \mathrm{W})$ carece de bienestar $\neg(\mathrm{C} \square \rightarrow \neg \mathrm{W})$. público

La conclusión del esquema $(\beta)$ implica

En nuestra propuesto, este esquema de fórmula es la oración: no es el caso que, si en la Isla Utopía no hay propiedad privada, entonces su sociedad no carece de bienestar público. En otras palabras, Moro niega el condicional que defiende Rafael compuesto por los enunciados "ser una sociedad sin propiedad privada", como antecedente, y "no carecer de bienestar público", como consecuente. La situación contrafáctica propuesta por el viajero no ha logrado llegar a buen puerto, al menos en lo que respecta al Moro-personaje.

En este sentido, en el pasaje problemático Moro-personaje propone otra lectura posible de la Isla Utopía, tan válida como la de Rafael. Con válido nos referimos a que es una instancia de otro esquema de argumento posible que también incluye en sus premisas al experimento mental. Moro-personaje, al ofrecer otra lectura posible que parte también de la misma situación contrafáctica, encarna la diversidad y complejidad propia de los procesos de interacción discursiva. Ahora bien, esta propuesta descansa, innegablemente, sobre una concepción polifónica de la obra. Abordamos este punto en la siguiente sección.

3.d-Algunos comentarios sobre los compromisos de nuestra lectura: Utopía y su carácter polifónico

Concebir a la Isla Utopía como una premisa contrafáctica dentro de dos argumentos que se van desplegando a lo largo de la obra requiere, previamente, la 
aceptación del carácter polifónico de la obra. El foco de la interpretación que propone Sánchez, siguiendo a Blaim, está puesto en entender a la obra como un corpus dialogístico de agencias que interactúan entre sí. Desde esta óptica, Moro-autor entiende que su visión del mundo social se encuentra enfrentada a muchas otras concepciones. Todas ellas inmersas en una red de interacciones, en donde cada una influye y modifica a las demás y, a su vez, es modificada e influenciada:

Tanto Moro como su personaje son conscientes de que la aprobación y el consenso, es decir la determinación práctica de la verdad de un enunciado, no depende, en este mundo, tanto de su contenido como de quién lo enuncia. El lenguaje es un instrumento para situarse en el grupo, por lo tanto, su uso se orienta mucho más a la conveniencia práctica del intercambio social que al descubrimiento de la "verdad" nada tiene un valor en sí, sino que "valor" es el resultado de un proceso en el que siempre hay dos agencias -o más- en mutua incidencia, de un proceso en el que lo que verdaderamente importa es el efecto práctico y la rentabilidad de los actos valorativos (Sánchez, 2011, p. 38).

En este sentido, el argumento de Moro-personaje y el argumento de Rafael son dos voces inmersas en este corpus dialogística. La obra, desde esta perspectiva, tiene un claro carácter polifónico. Al articular esta propuesta junto con el concepto de experimento mental, consideramos que hemos logrado desarticular algunos aspectos de Utopía que, en una primera lectura, parecerían ser problemáticos; aspectos relativos a la relación entre realidad y ficción. 


\section{4-Conclusiones}

En nuestro trabajo hemos tratado de argumentar a favor de una lectura de Isla Utopía en los términos de un experimento mental. Tal propuesta posiciona al relato de Rafael en el Libro Segundo como una descripción de una situación contrafáctica cuyo fin es constituirse como una premisa dentro de un argumento mayor. La hipótesis principal a refutar en dicho argumento es la idea de que el fundamento de todo orden social es la propiedad privada. Pero, siguiendo Häggqvist, no hay un único esquema de argumento relacionado con los experimentos mentales. Moro-personaje, en uno de los últimos pasajes, ejemplifica otra forma válida para interpretar el relato de Rafael; otro argumento posible que también incluye entre sus premisas a la situación contrafáctica. Desde esta lectura, Rafael y Moro-personaje construyen dos argumentos posibles, ambos válidos, pero con conclusiones diferentes. Rafael y Moro son dos voces, dos visiones del mundo que chocan pero que, a su vez, se influyen mutuamente, se modifican; dos concepciones del mundo social inmersa en una red de interacciones. Nuestra propuesta, por tanto, descansa sobre una visión polifónica de la obra. En este sentido, la lectura que ofrecemos de la Isla Utopía requiere, previamente, entender a la obra en los términos de un corpus dialogístico de agencias que interactúan unas con otras. Pero damos un paso más: la originalidad de nuestra propuesta reside en articular la visión polifónica con el concepto de experimento mental. Dicha noción nos permite presentar una propuesta renovada con mayor sistematización y solidez. 


\section{Bibliografía:}

Blaim, A. (1982): More's Utopia: persuasion or polyphony?, Moreana, 19 (73), pp.5-20.

Brown, J. R., (1991), Laboratory of the Mind: Thought Experiments in the Natural Sciences, London: Routledge.

Brown. J. y Fehige, Y., (1996), Thought Experiments, Stanford Encyclopedia of Philosophy: https://plato.stanford.edu/entries/thought-experiment/.

Häggqvist, S., (2009), A Model for Thought Experiments, Canadian Journal of Philosophy, 39, (1), pp. 56-76, DOI: https://doi.org/10.1353/cjp.0.0040.

Häggqvist, S., (1996) Thought Experiments in Philosophy, Stockholm: Almqvist \& Wiksell International.

Moro, T., (1987), Utopía, traducción y notas de E. García Estebanez, Madrid: Tecnos.

Norton, J. D., (1991), Thought Experiments in Einstein's Work, in T. Horowitz and G. Massey (eds.), Thought Experiments in Science and Philosophy, pp. 129-148, Lanham: Rowman \& Littlefield.

Prévost, A., (1969), L'Utopie de Thomas More, Paris: Mame.

Stuart, T., Fehige, Y. y Brown J., (eds.), (2018), The Routledge Companion to Thought Experiments, London and New York: Routledge.

Sánchez, J., (2011), Utopía e ironía en el contexto de Tomás Moro, Revista de Filosofía, 36, (1), p. 29.

Sylvester, S. (1977): Si hythlodaeo Credimus: Vision and Revision in Th. More's Utopie, R. S. Sylvester y G. P. Marcadow (ed.), Essential Articles for the study of Thomas More, pp. 290-30. , Hamdem: Archon Books. 
Abril 2021.

ISSN 2344-9934

Van Dyck, M., (2003), The Roles of One Thought Experiment in Interpreting Quantum Mechanics: Werner Heisenberg Meets Thomas Kuhn, Philosophica, 72, pp. $1-21$. 